\title{
Féeries
}

Études sur le conte merveilleuX, XVII ${ }^{e}$ XIXe siècle

Le conte, les savoirs

\section{Sous la cendre - Figures de Cendrillon, anthologie établie et postfacée par Nicole Belmont et Elizabeth Lemirre, Paris, Éd. José Corti, 2007, 423 p.}

\section{Anne Defrance}

\section{OpenEdition}

Journals

Édition électronique

URL : http://journals.openedition.org/feeries/723

DOI : $10.4000 /$ feeries.723

ISSN : 1957-7753

Éditeur

UGA Éditions/Université Grenoble Alpes

Édition imprimée

Date de publication : 1 juillet 2009

Pagination : 259-263

ISBN : 978-2-84310-140-3

ISSN : $1766-2842$

Référence électronique

Anne Defrance, « Sous la cendre - Figures de Cendrillon, anthologie établie et postfacée par Nicole Belmont et Elizabeth Lemirre, Paris, Éd. José Corti, 2007, 423 p. », Féeries [En ligne], 6 | 2009, mis en ligne le 15 septembre 2010, consulté le 10 décembre 2020. URL : http://journals.openedition.org/ feeries/723; DOI : https://doi.org/10.4000/feeries.723 


\section{COMPTE RENDU CRITIQUE}

Sous la cendre - Figures de Cendrillon, anthologie établie et postfacée par Nicole Belmont et Elizabeth Lemirre, Paris, Éd. José Corti, 2007, 423 p.

Sont réunies dans cette anthologie quarante-sept versions d'un conte universellement connu, grâce surtout à Perrault et aux frères Grimm ${ }^{\mathrm{I}}$ chez les Occidentaux, sous le nom de Cendrillon. Nicole Belmont a consacré déjà plusieurs articles à cette "figure mythique ", comme elle la désigne à la suite des Grimm, dans sa Poétique du conte (1999, p. 197).

Il a donc bien fallu faire un choix dans les centaines de versions recensées par les ethnologues et folkloristes. Elles sont classées en cinq sections : France, Europe, Afrique, Asie, Amérique (cette dernière incluant des versions québécoise et guadeloupéenne). En fin de volume, le lecteur trouvera une bibliographie des sources utilisées par les deux éditrices, ainsi qu'une table alphabétique et typologique.

Dans une brève préface intitulée "Le cycle de Cendrillon », N. Belmont, utilisant la typologie internationale Aarne-Thompson-Uther, explique les critères de sélection des versions : n'ont été retenus que les sous-types T sio A ("Cendrillon/Cinderella »), T siI (« Un-CEil, Deux-Yeux, TroisYeux / One-Eye, Two-Eyes, Three-Eyes »). Ont été exclus deux autres, le T sio B ("Peau d'Âne / Peau d'Asne »), forme " mutante » et plus récente " qui tend vers le romanesque littéraire " (p. 8) - on comprend donc qu'il intéresse moins la spécialiste de tradition orale qu'elle est - et le T 5II B ( Le Petit Bœuf rouge »), dont le héros incarne la variante masculine de l'héroïne - mais nous ne saurons rien des raisons de cette mise à l'écart.

I. Signalons au passage que le numéro 4 de Féeries, consacré aux rapports entre conte et scène, a ouvert ses pages à d'autres Cendrillon : Noémie Courtès, "Cendrillon mise en pièces ou la seconde immortalité de Perrault au XIX ${ }^{e}$ siècle »; Benjamin Pinthiaux, "Hippolite et Cendrillon »; Isabelle Degauque, «Des contes des Mille et Une Nuits à La Pantoufle de Marignier (1730) ». 
Le conte a fait le tour du globe jusqu'aux Antilles, de l'Occident vers l'Orient, en laissant de côté l'Afrique noire. Des deux ouvrages qui en ont répertorié les versions à partir de la fin du XIX ${ }^{e}$ siècle, $\mathrm{N}$. Belmont retient tout particulièrement celui d'A. B. Rooth (The Cinderella cycle, I95I), qui a remanié leur typologie, suivant leur structure narrative, pour en dégager six types et parvenir à l'hypothèse d'une évolution dans l'espace et le temps. Mais l'auteur émet ici quelques réserves, pour la raison que des récits de type différents coexistaient dans les mêmes régions. Elle conteste ensuite la thèse de Graham Anderson, qui en 2000, a cru trouver des témoignages de l'existence de ce récit dans l'Antiquité, chez Strabon ( $\mathrm{I}^{\text {er }}$ siècle av. et apr. J.-C.). Le conte vient donc du Moyen-Orient, même si sa première forme écrite, datant $\mathrm{du}_{\mathrm{IX}} \mathrm{e}^{\mathrm{e}}$ siècle, est chinoise. Au terme de cette présentation récapitulative et critique, elle propose un mode de lecture des versions de Cendrillon, des plus simples aux plus complexes, suivant un ordre - ou plutôt un sens - qui ne correspond pas à l'ordre géographique de leur édition dans cette anthologie, choisi à défaut d'un mouvement chronologique puisqu'on ne dispose d'aucun moyen de dater l'apparition de récits appartenant à la tradition orale. Il s'agirait donc les lire " comme un itinéraire psychique, celui des filles face à leur mère [...], qui doit aboutir à l'acquisition de la féminité, à la conscience de sa propre identité, et à la reconnaissance de celle-ci »(p. I2). Cette lecture est, on le voit, conforme dans ses conclusions à celle d'autres critiques inspirés comme elle par la psychanalyse. Une question peut alors se poser au lecteur : considéré dans ses grandes lignes, cet itinéraire psychique est-il l'exclusivité de Cendrillon ? N'est-il pas partagé par d'autres héroïnes de contes? Osons même avouer une seconde question qui nous est venue à l'esprit à ce moment de la lecture : est-il l'apanage du féminin ? Il suffirait peut-être de masculiniser les termes de la précédente citation... Il est vrai que ces récits écartés sans raison apparente de l'anthologie, les versions du type $\mathrm{T}$ 5II B, titillent la curiosité : il faut bien que le refoulé fasse un peu retour. Mais ces questions sont aussi évidemment induites par l'inévitable schématisme inhérent à la récapitulation de cette trajectoire, comme à toute vue surplombante. Formant diptyque et placés en postface, les deux essais proposés par les éditrices de ces récits y répondront.

Dans le premier, N. Belmont montre comment la nébuleuse du cycle de Cendrillon est bien " une affaire de femmes" (p. 363-379), conformément à son titre, formulé à la forme interrogative. D'un récit du cycle à l'autre, et à l'aide de citations nombreuses, elle effectue ce parcours qu'elle avait, à l'orée de l'ouvrage, invité son lecteur à entreprendre. Elle démontre - lumineusement - que le cycle entier opère bel et bien la « mise en récit 
de la maturation psychique de la jeune fille» (p. 363). Elle part, naturellement, de ceux qui expriment les pulsions les plus archaïques, et de façon parfois sauvage (gageons qu'ils surprendront le lecteur qui ne connait que les versions classiques et littéraires présentant une Cendrillon orpheline de mère, ce qui n'est pas du tout systématique dans les versions populaires). Récits, tout d'abord, où la pulsion orale, exprimant le lien primaire unissant la mère à ses enfants, est mise en scène, lien " que le récit va s'efforcer d'épurer et de poétiser » (p. 364). Elle évoque ensuite les récits dans lesquels l'héroïne tue sa mère, sur incitation d'une autre femme le plus souvent. Ainsi dans la version corse, la mère est-elle littéralement coupée en deux parties par sa fille, laissant place à une sainte et une vache (sous laquelle transparaît symboliquement la nourrice idéale). Ce « dédoublement en deux effigies opposées [...] réitère celui qu'instaure la partition entre une mère aimante et absente et une mère malveillante et présente : clivage fondamental dans cette histoire» (p. 377). L'auteur montre aussi comment chacun à leur manière d'autres récits, plus élaborés, illustrent le passage de l'oralité à la sexualité, « de la nourriture aux parures » qui feront prendre à la jeune fille conscience de sa beauté, la sortiront des cendres - figures du deuil de la mère - et la mèneront à l'amour².

Dans l'autre volet du diptyque, Elizabeth Lemirre tourne son attention, quant à elle, "Du côté des hommes ». Mais il ne s'agit toujours pas d'évoquer les versions masculines exclues de l'ouvrage : c'est la relation de l'héroïne au père qui est tout d'abord explorée ici. Père qui n'est pas si absent qu'il le paraît. Il persiste symboliquement, montre-t-elle, dans le foyer où Cendrillon est recluse, quand il n'est pas déplacé dans le personnage de l'étranger, pourvoyeur d'habits - ici accompagnateur de l'héroïne, là mendiant jouant un rôle également salvateur. Dans d'autres versions, le père est présent effectivement. Remarié, il a coupé le lien avec sa fille, dominé qu’il est par les autres figures féminines, marâtre ou sœurs. Père vaguement incestueux, dans d'autres récits encore. Peau d'Âne fuit pour se dégager du désir de son père, mais à l'inverse, Cendrillon, ensevelie dans les cendres (de sa mère), ne peut si facilement les quitter pour aller vers un autre objet d'amour, celui qui fera d'elle une femme. Là réside en partie l'originalité de cette lecture, ce me semble, lecture toujours inspirée

2. L'interprétation de ce motif est différente chez Bernadette Bricout (La clé des contes, Seuil, 2005). Elle relie les cendres à une autre tâche ménagère dans lesquelles Cendrillon est confinée, et qu'on a tendance à oublier selon elle, la lessive (voir notre compte rendu dans Féeries, $n^{\circ} 5$, p. 153 ). Nous remarquons que cette tâche n'apparaît d'ailleurs que dans une seule des versions françaises retenues dans cette anthologie (la version corse, Cinnareda), mais qu'elle est davantage présente dans les versions étrangères. 
par la psychanalyse, même si les outils n'en sont pas exhibés (car il s'agit bien ici du complexe d'Edipe - mais sont évitées ici les schématisations doctrinales) : Cendrillon est vue comme désirant le père, elle se macule pour s'en faire, paradoxalement, mieux voir et après s'être " défigurée ", va se "refigurer d'une autre façon" (p. 390). Cela éclaire l'épreuve du tri des graines, mélangées aux cendres dans de nombreuses versions : à la jeune fille, alors, de " séparer ce qui est du côté de la vie (les graines) de ce qui est du côté de la mort (la mère qu'elle porte en elle) » (p. 39I). L'essai s'achève sur une autre interprétation tout aussi séduisante, qui prolonge celle de Nicole Belmont, qui reconnaît en Cendrillon, à la suite de Jean-Pierre Vernant, une héritière d'Hestia. Il n'y manquait plus que son pendant, Hermès. E. Lemirre l'a retrouvé, masqué sous la figure du prince qui fait, selon elle, de cette femme transie une femme en mouvement et qui a, lui aussi, une quête à accomplir, celle d'une épouse, suivant son propre itinéraire. Il tient dans sa main, fasciné, cette pantoufle, la chaussure (sans les ailes) que le dieu a perdue : "Cendrillon dirait alors le dernier transfert d'une femme par Hermès et la résignation du divin à devenir mortel, comme un pendant à la destination d'Hestia en Cendrillon. Il se jouerait donc, dans Cendrillon, un adieu aux dieux " (p. 396). Difficile de résister à une telle lecture, qui illustre la définition que Lévi-Strauss donnait du conte (un mythe désacralisé).

Serait-ce aller trop loin de que de voir alors dans la figure de Cendrillon, à partir des deux lectures complémentaires ici présentées, non seulement celle de l'évolution de la féminité, mais encore l'emblème de l'histoire de ces récits, histoire enfouie "sous la cendre" (histoire recomposée, refigurée, puisque les repères chronologiques sont insaisissables) et, plus encore, l'emblème de celle des contes en général (sans prétendre pour autant bien sûr que le conte de Cendrillon s'en approprie l'exclusivité) ?

Il est impossible de rendre compte dans un résumé de toutes les finesses de ces deux interprétations, suggestives et toujours convaincantes, même si l'exhumation du fil souterrain qui représente une abstraction, l'itinéraire psychique de l'héroïne, a parfois induit la mise à l'écart d'éléments concrets qui ne vont pas dans le sens de la thèse. Par exemple, l'impulsion donnée à l'héroïne ne vient pas toujours explicitement d'un homme (le prince ou son père, voire un inconnu comme dans La Cendrouse), même s'il est difficile de nier que c'est un homme, prince ou roi, qui " met en branle la société »(E. Lemirre, p. 395). Dans Datte, belle date et Cinnareda, ainsi que Cendrouzette, le rôle moteur échoit pourtant à une femme, fée ou belle dame, ou encore à un animal merveilleux dans Petite anguille, Maroula, etc. À moins même que l'impulsion ne vienne, plus souterrai- 
nement, de la répression du désir, de l'interdit de quitter la maison pour aller au bal. Qui suscite alors le mouvement ? La marâtre, qui défend toute sortie, celui qui part en quête d'une épouse ou organise le bal, les sœurs qui s'y rendent, celui ou celle qui délivre le moyen de détourner cet interdit ? On pourrait sans doute ajouter d'autres instances à la liste, et débattre de la question pour faire ressortir celui qui serait le bon ou le plus décisif - ils le sont tous, suivant la perspective choisie.

Ajoutons enfin que l'écriture de ces essais séduit aussi par la poésie dont ils sont parfois emprunts. Le dernier est d'ailleurs placé sous l'exergue d'une jolie citation de Pascal Quignard (Sur le jadis), où il est question de cette " friche d'enchantement " que proposent les contes. Renvoyons donc le lecteur à son propre défrichage/déchiffrage de ces essais et, bien sûr, des récits qu'ils analysent. Dans leur riche diversité, ces derniers font entendre le grain de la voix des conteurs, tantôt âpre, tantôt plus veloutée. Les formes dialectales, ces aspérités les plus obscures de leur retranscription, sont éclairées par un appareil de notes lexicographique suffisant, jamais gênant (même si - mais c'est un détail - les notes 2 et I4 de Cendrouzette font un peu froncer le sourcil).

Anne Defrance 